\title{
Medición del comportamiento social individual y percepción de miedo durante la pandemia por COVID-19 en jefes de familia
}

\author{
Measurement of individual social behaviour and perception of fear during the pandemic by COVID-19 in \\ heads of households \\ Medição do comportamento social individual e percepção de medo durante a pandemia por COVID-19 \\ em chefes de família
}

\section{Resumen}

Objetivo: Identificar las características del comportamiento social e individual y percepción de miedo durante la pandemia COVID-19 en jefes de familia en aislamiento social. Material y métodos: Estudio observacional de corte transversal, prospectivo y analítico. Se incluyó a 372 jefes de familia residentes del cercado de Tacna durante el año 2020, seleccionados por muestreo multietápico; se excluyó extranjeros, transeúntes, personas en aislamiento obligatorio por infección COVID-19 por caso confirmado o contacto sospechoso y personas que no adoptaron medidas básicas de bioseguridad. Se aplicó cuestionario de medición de percepción de comportamiento social y miedo, en domicilio previo consentimiento informado. Se utilizó estadística descriptiva y analítica con regresión logística para determinar fuerza de asociación con análisis multivariante. Resultados: Los jefes de familia se caracterizaron por tener edades entre 40 a $49(37,6 \%)$, convivientes $(65,1 \%)$, secundaria completa $(51,1 \%)$. El 8,3\% tuvo contacto con familiar sospechoso con COVID-19 bajo el mismo techo, el 34,9\% con familiar cercano. Considera riesgo de padecer COVID-19 $(43,5 \%)$. Respecto a conducta social, el $89,5 \%$ adopta comprar mascarillas respiratorias; el 81,7 $\%$ adquisición de alcohol; solo el $39 \%$ adopta el lavado de manos. El 54,6\% ha cumplido con aislamiento parcial, el 32,8\% prefiere no opinar y solo el 12,7 \% realizó aislamiento total permanente. Conclusión: Existen diferencias significativas según edad y condición de contacto con compañeros de trabajo asociados al miedo. El comportamiento social fue estadísticamente diferente según edad, el entorno de riesgo es principalmente por compañeros de trabajo.

Palabras clave: comportamiento social, comportamiento individual, temor, miedo, COVID-19

\section{Abstract}

Objective:To identify the characteristics of social and individual behavior and perception of fear during the COVID-19 pandemic in socially isolated heads of households. Material and methods: Cross-sectional, prospective and analytical observational study. 372 heads of households resident in Tacna city were included, during the year 2020, selected by multistage sampling; foreigners, bystanders, persons in mandatory isolation due to COVID-19 infection were excluded per confirmed case or suspicious contact and persons who did not take basic biosecurity measures. We applied a

'Centro de Salud Tarata, Ministerio de Salud. Médico cirujano. Tacna, Perú

Universidad Nacional Jorge Basadre Grohmann. Médico cirujano. Doctor en Salud Pública. Docente de la Escuela de Medicina Tacna, Perú 
questionnaire to measure the perception of social behavior and fear in the home with informed consent. Descriptive and analytical statistics with logistic regression were used to determine strength of association with multivariate analysis. Results: The heads of families were characterized by having ages between 40 and 49 (37.6\%), cohabiting (65.1\%), complete high school (51.1\%). $8.3 \%$ had contact with suspected family members with COVID-19 under the same roof, $34.9 \%$ with close family members. Consider the risk of developing COVID-19 (43.5\%). Regarding social behavior, $89.5 \%$ adopt buying respiratory masks; $81.7 \%$ purchase alcohol; only $39 \%$ adopt hand washing. $54.6 \%$ had partial insulation, $32.8 \%$ preferred not to have an opinion and only $12.7 \%$ had permanent total insulation. Conclusion: There are significant differences according to age and condition of contact with coworkers associated with fear. Social behavior was statistically different according to age, the environment of risk is mainly by co-workers.

Keywords: social behavior, individual behavior, fear, COVID-19

\section{Resumo}

Objetivo: Identificar as características do comportamento social e individual e percepção de medo durante a pandemia COVID-19 em chefes de família em isolamento social. Material e métodos: Estudo observacional de corte transversal, prospectivo e analítico. Foram incluídos 372 chefes de família residentes do cercado de Tacna durante o ano 2020, selecionados por amostragem multietápica; foram excluídos estrangeiros, transeuntes, pessoas em isolamento obrigatório por infecção COVID-19 por caso confirmado ou contacto suspeito e pessoas que não tomaram medidas básicas de biossegurança. Aplicou-se questionário de medição de percepção de comportamento social e medo, em domicílio prévio consentimento informado. Utilizou-se estatística descritiva e analítica com regressão logística para determinar a força de associação com análise multivariante. Resultados: Os chefes de família caracterizaram-se por terem idades entre 40 e 49 (37,6\%), conviventes (65,1 $\%)$, secundário completo (51,1\%). 8,3\% tiveram contato com parentes suspeitos com COVID-19 sob o mesmo teto, 34,9\% com parentes próximos. Considera-se que existe risco de COVID-19 (43,5 \%). No que se refere ao comportamento social, $89,5 \%$ dos consumidores compram máscaras respiratórias; 81,7 \% adquirem álcool; apenas $39 \%$ adotam a lavagem das mãos. Os 54,6 \% cumpriram com isolamento parcial, 32,8 \% preferem não opinar e apenas $12,7 \%$ fizeram isolamento total permanente. Conclusão: Existem diferenças significativas segundo idade e condição de contato com colegas de trabalho associados ao medo. O comportamento social foi estatisticamente diferente segundo a idade, o ambiente de risco é principalmente por colegas de trabalho.

Palavras-chave: comportamento social, comportamento individual, medo, medo, COVID-19

\section{Introducción}

En la actual pandemia por coronavirus, iniciada en China en diciembre de 2019, el $81 \%$ de los casos ha desarrollado neumonía, con una tasa de mortalidad promedio de 2,3 \%.' La Organización Mundial de la Salud, el 11 de marzo de 2020, casi 90 días después de la aparición del primer caso, y debido a su alta propagación, el número de casos y la gravedad clínica refiere que, además de todo lo clínico conocido no se puede ignorar su impacto psicológico. Considerando que es una patología reciente, ha surgido una variada investigación sobre el estrés emocional en la situación actual.

Las sociedades modernas están expuestas a una miríada de riesgos que van desde enfermedades hasta peligros naturales e interrupciones tecnológicas. ${ }^{3}$ Cuando enfrentan riesgos, las personas pasan por un proceso complejo de recopilación de información, decidir qué hacer y comunicarse con otros sobre la efectividad de sus acciones. La influencia social puede interferir con las experiencias personales, lo que hace que los grupos de pares y las interacciones grupales 
sean factores importantes. Esto es especialmente importante para comprender la difusión de la enfermedad y la aparición de epidemias. ${ }^{4}$ Por lo tanto, las buenas estrategias de respuesta y prevención tanto a nivel individual como gubernamental son vitales para salvar vidas. La elección de una estrategia depende de los aspectos conductuales, las interacciones complejas entre las personas ${ }^{5}$ y la información disponible sobre una enfermedad. ${ }^{6}$ Percibir el riesgo de una enfermedad infecciosa puede desencadenar un cambio de comportamiento, como durante la epidemia de SARS de 2003. Recopilar información y experiencia a través de múltiples fuentes es esencial para aumentar la conciencia sobre el riesgo de enfermedad y tomar medidas de protección.

Dos procesos son esenciales para representar el comportamiento de salud de los agentes y la dinámica de la enfermedad, la evolución de la percepción del riesgo y la selección de una estrategia de afrontamiento. Por lo tanto, el núcleo de una enfermedad $A B M$ radica en definir los métodos de aprendizaje que dirigen estos dos procesos. La detección de información (global, del entorno y social, es decir, de otros agentes), el intercambio de información (es decir, las interacciones entre agentes) y el procesamiento de la información (es decir, la toma de decisiones) son críticos. Las técnicas de aprendizaje automático $(\mathrm{ML})$ pueden respaldar estos tres elementos y ofrecer una forma más realista de ajustar el comportamiento de los agentes en $\mathrm{ABM}{ }^{9,10}$

Los agentes, individuos y grupos, pueden aprender de forma aislada o mediante interacciones con otros, como sus vecinos. En el aprendizaje aislado, los agentes aprenden de forma independiente y no requieren interacción con otros agentes. En el aprendizaje interactivo, varios agentes se dedican a detectar y procesar información y a comunicarse y cooperar para aprender de manera efectiva. El aprendizaje interactivo se puede realizar de múltiples maneras, es decir, en base a diferentes estrategias de aprendizaje social."
La estigmatización y la culpa han estado relacionadas desde hace mucho tiempo con epidemias. Es bien sabido que la Peste Negra provocó la persecución masiva de judíos en varias partes de Europa, ${ }^{12}$ la pandemia del VIH/SIDA de la década de 1980 provocó un prejuicio extremo contra los homosexuales y los usuarios de drogas intravenosas. ${ }^{13}$ La globalización y el temor de que las enfermedades exóticas puedan transportarse a los entornos urbanos modernos ${ }^{14}$ ha aumentado en cierta medida este tipo de preocupaciones sobre los chivos expiatorios. Los virus no conocen fronteras $y$, por lo tanto, se enredan fácilmente con las ansiedades contemporáneas sobre la migración y los refugiados. ${ }^{15}$ Las poblaciones asiáticas en los barrios chinos de varias ciudades occidentales fueron víctimas del síndrome respiratorio agudo severo (SRAS) ${ }^{16}$ y los estudios realizados en Hong Kong revelaron que el público realmente anticipó este tipo de resultado."

Aunque a menudo se reconoce que los humanos toman medidas preventivas en el curso de una epidemia, los modelos que incorporan dinámicas de comportamiento son generalmente mucho más difíciles de analizar. Recientemente, estos modelos han comenzado a recibir más atención, y se han realizado importantes avances para comprender el efecto de los diferentes cambios de comportamiento en la dinámica de la epidemia. ${ }^{18}$ Dado el comportamiento epidemiológico de COVID-19, puede requerirse un período indefinido de aislamiento social; independientemente de la pandemia, el aislamiento social en sí mismo puede causar síntomas psicóticos. ${ }^{19}$

Nuestra realidad, no escapa a la vista a nivel mundial, padeceremos similares estados de estrés poblacional. El objetivo de este estudio fue identificar las características del comportamiento social e individual y la percepción de miedo durante la pandemia COVID-19 en jefes de familia en aislamiento social en el cercado de Tacna. 


\section{Material y métodos}

Estudio observacional de corte transversal, prospectivo y analítico. De la población residentes del cercado de Tacna, se seleccionó aleatoriamente una muestra de 372 jefes de familia, según el mapa de distribución por manzanas, los edificios multifamiliares fueron considerados como una manzana. Se solicitó consentimiento y participación voluntariamente. Se excluyó extranjeros, transeúntes, personas en aislamiento obligatorio por infección COVID-19 por caso confirmado o contacto sospechoso y personas que no adoptaron medidas básicas de bioseguridad (mascarilla y distanciamiento mayor a 1 metro). El muestreo fue por conglomerados multietápicos.

Se entrevistó y aplicó instrumento de medición de percepción de comportamiento social y miedo, validado por Mejía, ${ }^{20}$ por su aplicabilidad regional y respaldado por publicaciones científicas, de las cuales se ajustó para fines de validación regional por Agüero. ${ }^{21}$ Se trabajó con grupo piloto para afinamiento del instrumento y se consignó una validación estadística mediante alfa de Cronbach aceptando un valor mínimo general y por cada pregunta seleccionada de 0,8 y se sometió a juicio de expertos: salubristas (2), psiquiatras (2), infectólogo y/o intensivistas que manejaron pacientes y familiares COVID-19 confirmados (1), médicos Internistas (2) que manejaron pacientes confirmados y sospechosos COVID19 (2). El Instrumento inicial propuesto partió de la opinión de equipo de asesoría profesional de personal médico en manejo de pacientes sospechosos y/o confirmados COVID-19o estén dirigiendo el control de la epidemia a nivel regional.

Se consideró la escala de Likert para la medición con valores de 1 a 5, considerándose las características en total desacuerdo a muy de acuerdo. Los instrumentos fueron aplicados por un equipo de entrevistadores, previa capacitación quienes realizaron una visita domiciliaria.

Los resultados se presentan en tablas y figuras de doble entrada. Se utilizó pruebas univariadas y bivariadas para determinar las principales variables relacionadas calculando valores absolutos y relativos. Se realizó regresión logística para determinar fuerza de asociación con análisis multivariante. Todos los cálculos consideraron un nivel de confianza del $95 \%$, con unvalor p significativo menora 0,05.

Se guardó absoluta confidencialidad de la identidad de los participantes, la información fue totalmente anónima. Los resultados se manejaron absolutamente con fines académicos y de investigación.

\section{Resultados}

Los jefes de familia participantes en este estudio se caracterizaron por tener $37,6 \%$ de 40 a 49 años, $21,5 \%$ de 50 a 59 años y 15,6\% entre 30 a 39 años; $65,1 \%$ tenía la condición de conviviente y el $26 \%$ de casado(a), 51,1 \% tenía nivel de secundaria completa, seguido de un $25 \%$ con nivel técnico y el $18,5 \%$ con nivel superior.

Si analizamos estas características sociodemográficas asociadas a miedo en el contexto de la pandemia, observamos que en el grupo de 20 a 29 años se encuentra una diferencia estadísticamente significativa, que confirma que el miedo es muy bajo o ausente $(p<0,01)$ comparado al resto de grupos de edad, considerándose como de riesgo y que se sabe con características de transmisibilidad alta en la comunidad en la cual deambula. Los otros grupos que consideran un estado de presencia de mayor miedo es en los grupos de 50 años a más. Estas diferencias son altamente significativas $(p<0,01)$. No hubo diferencias según el estado civil o el nivel de instrucción (Tabla 1). 
Tabla 1

Características sociodemográficas asociadas a miedo en el contexto de la pandemia

\begin{tabular}{|c|c|c|c|c|c|}
\hline $\begin{array}{l}\text { Características del } \\
\text { jefe de familia }\end{array}$ & $\mathrm{n}=372$ & $\%$ & $\begin{array}{c}\text { Error } \\
\text { estándar }\end{array}$ & Wald & $\mathbf{p}$ \\
\hline \multicolumn{6}{|l|}{ Edad } \\
\hline De 20 a 29 años & 38 & 10,2 & & 21,363 & 0,000 \\
\hline De 30 a 39 años & 58 & 15,6 & 0,567 & 3,847 & 0,050 \\
\hline De 40 a 49 años & 140 & 37,6 & 0,456 & 3,243 & 0,072 \\
\hline De 50 a 59 años & 80 & 21,5 & 0,458 & 20,16 & 0,000 \\
\hline Más de 60 años & 56 & 15,1 & 0,446 & 8,387 & 0,004 \\
\hline \multicolumn{6}{|l|}{ Estado civil } \\
\hline Casado & 97 & 26,1 & & 7,606 & 0,055 \\
\hline Conviviente & 242 & 65,1 & 20095,53 & 0,000 & 0,999 \\
\hline Separado/divorciado & 29 & 7,8 & 20095,53 & 0,000 & 0,999 \\
\hline Viudo & 4 & 1,1 & 20095,53 & 0,000 & 0,999 \\
\hline \multicolumn{6}{|l|}{ Nivel de instrucción } \\
\hline Analfabeto & 1 & 0,3 & & 5,671 & 0,225 \\
\hline Primaria & 17 & 4,6 & 40192,97 & 0,000 & 1,000 \\
\hline Secundaria & 190 & 51,1 & 0,875 & 2,579 & 0,108 \\
\hline Técnico & 95 & 25,5 & 0,425 & 0,012 & 0,911 \\
\hline Superior & 69 & 18,5 & 0,441 & 0,988 & 0,320 \\
\hline
\end{tabular}

Se encontró que el 8,3 \% refiere que tuvo contacto con familiar con COVID-19 bajo el mismo techo; el 34,9\%, con familiar cercano pero que no habitaba en la misma casa; el 28,2 \% refiere que supo de un amigo cercano que era COVID-19 positivo; el 19,1\%, un vecino; un 59,4\% desconoce si tuvo contacto con algún caso sospechoso. Los participantes de este estudio no manifiestan que la percepción de riesgo y miedo actual estén asociados a la posibilidad de que algún familiar, amigo, vecino o conocido cercano haya sido detectados anteriormente como sospechosos. La condición actual de temor no está condicionada por la percepción de la ocurrencia probable de detección anterior. En el análisis de los factores asociados se encontró que la característica "compañeros de trabajo" es la variable más asociada a la percepción de miedo y riesgo de contagio de COVID-19 en cualquier momento $(p=0,006)$ (Tabla 2). 
Medición del comportamiento social individual y

percepción de miedo durante la pandemia por COVID-19 en

jefes de familia

Tabla 2

Contacto sospechoso con COVID-19 en jefes de familia en aislamiento social en el cercado de Tacna

\begin{tabular}{|c|c|c|c|c|c|c|c|c|c|c|}
\hline \multirow{2}{*}{$\begin{array}{l}\text { Percepción de } \\
\text { riesgo por } \\
\text { conducta del } \\
\text { entorno }\end{array}$} & \multicolumn{2}{|c|}{ Sí } & \multicolumn{2}{|c|}{ No } & \multicolumn{3}{|c|}{$\begin{array}{c}\text { Percepción de tener } \\
\text { familiar/amigo sospechoso }\end{array}$} & \multicolumn{3}{|c|}{$\begin{array}{l}\text { Percepción de ser } \\
\text { contagiado por } \\
\text { familiar/amigo }\end{array}$} \\
\hline & N..$^{\circ}$ & $\%$ & $\mathrm{~N} .^{\circ}$ & $\%$ & $\begin{array}{c}\text { Error } \\
\text { estándar }\end{array}$ & Wald & $p$ & $\begin{array}{c}\text { Error } \\
\text { estándar }\end{array}$ & Wald & $\mathbf{p}$ \\
\hline $\begin{array}{l}\text { Familiar bajo el } \\
\text { mismo techo }\end{array}$ & 31 & 8,3 & 341 & 91,7 & 0,563 & 0,219 & 0,640 & 0,482 & 1,181 & 0,277 \\
\hline Familiar cercano & 130 & 34,9 & 242 & 65,1 & 0,303 & 0,597 & 0,440 & 0,294 & 0,455 & 0,500 \\
\hline Amigo & 105 & 28,2 & 267 & 71,8 & 0,353 & 3,173 & 0,075 & 0,302 & 0,016 & 0,900 \\
\hline Vecino & 71 & 19,1 & 301 & 80,9 & 0,371 & 0,014 & 0,907 & 0,301 & 2,325 & 0,127 \\
\hline Conocido & 151 & 40,6 & 221 & 59,4 & 0,284 & 0,159 & 0,690 & 0,283 & 7,667 & 0,006 \\
\hline
\end{tabular}

El $96 \%$ de la muestra en estudio usa principalmente la televisión como medio orientador sobre temas de COVID-19, seguido de un $71 \%$ de la radio, el 60,5\% a través de medios de internet, el 37,6\% a través de amigos y el 36,8 \% a través de periódicos.
El $43,5 \%$ asume que por conductas propias se considera en riesgo de padecer en cualquier momento de COVID-19. El 88,2\% percibe mayor riesgo de contagio por familiar que vive bajo el mismo techo; el $61 \%$ de familiar cercano o por compañero de trabajo, respectivamente; el 50,3 $\%$ a través de amigos y el $42,7 \%$ a través de contacto con vecinos (Tabla 3).

Tabla 3

Percepción de riesgo por conducta del entorno en jefes de familia en aislamiento social en el cercado de Tacna

\begin{tabular}{lcccccc}
\hline \multirow{2}{*}{ Percepción de riesgo de contagio } & \multicolumn{2}{c}{ Sí } & \multicolumn{2}{c}{ No } & \multicolumn{2}{c}{ Total } \\
\cline { 2 - 7 } & $\mathbf{N} .^{\circ}$ & $\%$ & $\mathbf{N}^{\circ}$ & $\%$ & N. ${ }^{\circ}$ & $\%$ \\
\hline Riesgo de contagio por conducta & 162 & 43,5 & 210 & 56,5 & 372 & 100 \\
propia & & & & & & \\
Riesgo de contagio por conducta & & & & & & \\
del entorno & 328 & 88,2 & 44 & 11,8 & 372 & 100 \\
Familiar bajo el mismo techo & 227 & 61 & 145 & 39 & 372 & 100 \\
Familiar cercano & 159 & 42,7 & 213 & 57,3 & 372 & 100 \\
Amigo & 187 & 50,3 & 185 & 49,7 & 372 & 100 \\
Vecino & 227 & 61 & 145 & 39 & 372 & 100 \\
Conocido & & & & & & \\
\hline
\end{tabular}

Respectoalas medidas de comportamiento social, en la dimensión de medidas de higiene y adquisitivas, observamos que el $89,5 \%$ adopta comprar mascarillas respiratorias, el 81,7\% adopta adquisición de alcohol, solo el $39 \%$ adopta el lavado de manos como una buena conducta (Figura 1). 


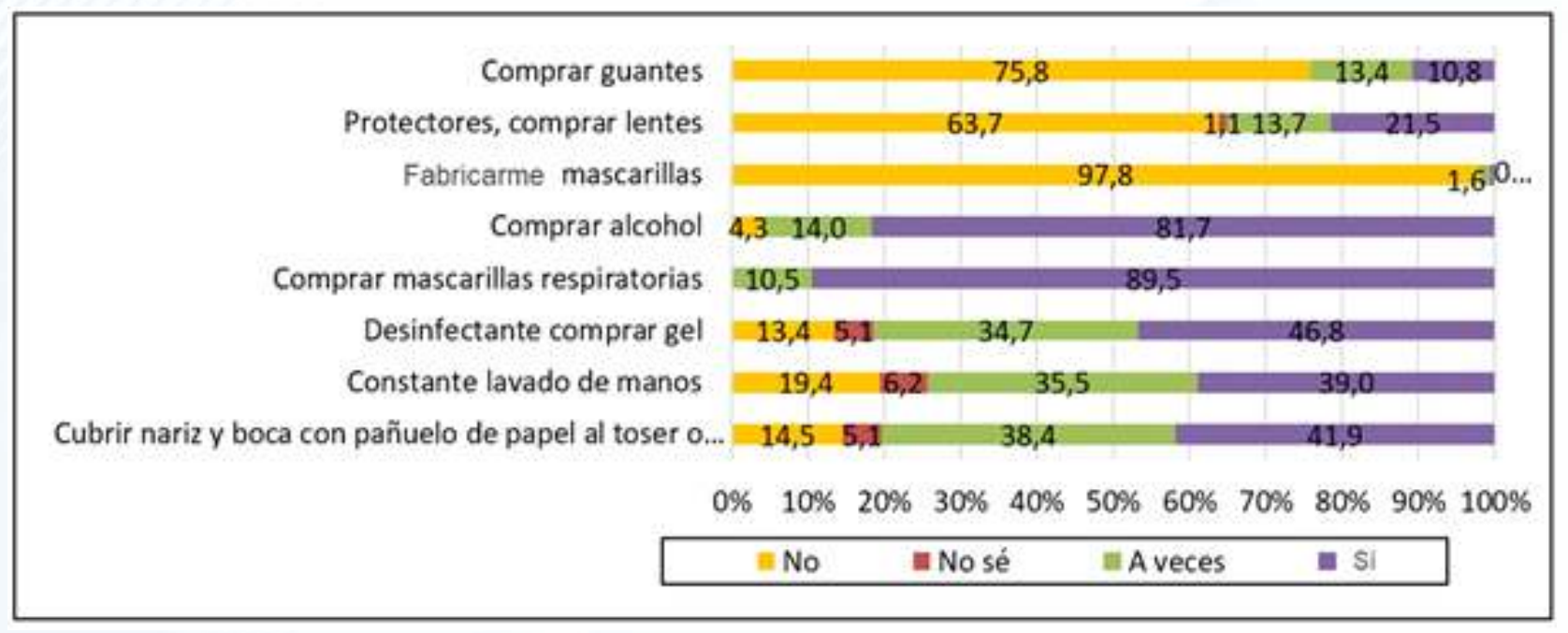

Figura 1. Medidas de higiene y adquisitivas del comportamiento social en jefes de familia en aislamiento social en el cercado de Tacna

Respecto a las medidas evitativas y de aislamiento, el 87,9\% refiere como una medida adecuada evitativa el uso de mascarillas en forma diaria, el 69,4\% el evitar visitar hospitales y centros de salud, el 47 \% evitar lugares públicos con mucha gente y un 51,1\% evitar los transportes públicos con mucha gente. Solo el
$58,3 \%$ refirió que el contacto con personas con gripe debería ser una adecuada medida evitativa. Respecto al aislamiento, el 54,6\% refiere que ha cumplido con este mecanismo parcialmente, el $32,80 \%$ prefiere no opinar al respecto y solo el $12,7 \%$ confirma haber realizado aislamiento total (Figura 2).

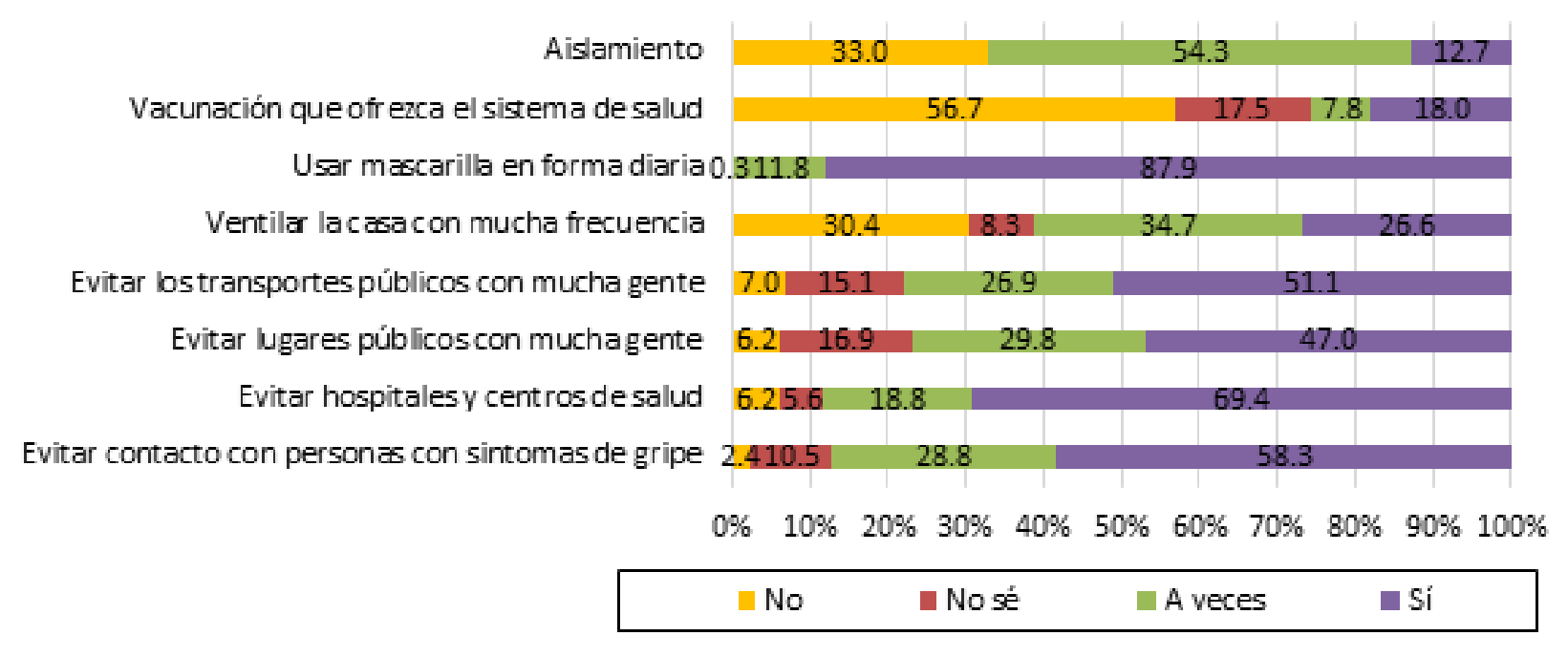

Figura 2. Medidas evitativas y de aislamiento en jefes de familia en aislamiento social en el cercado de Tacna

En la Figura 3, se observa la comparación de los diferentes estados o características del miedo en la actual condición de pandemia. El uso de la televisión como instrumento orientador genera mucho miedo seguido por la radio y la comunicación exagerada de la magnitud que realizan las emisoras locales. Considera como estados que también tienen una relación directa con el miedo actual, el uso de redes sociales y la exageración de la comunicación que en esos medios discurre. La población objeto de estudio considera que las características asociadas al temor fueron la comunicación recibida de familiares y amigos y la exageración que pueda haber existido en contadas ocasiones en los periódicos y diarios a los cuales acceden. 


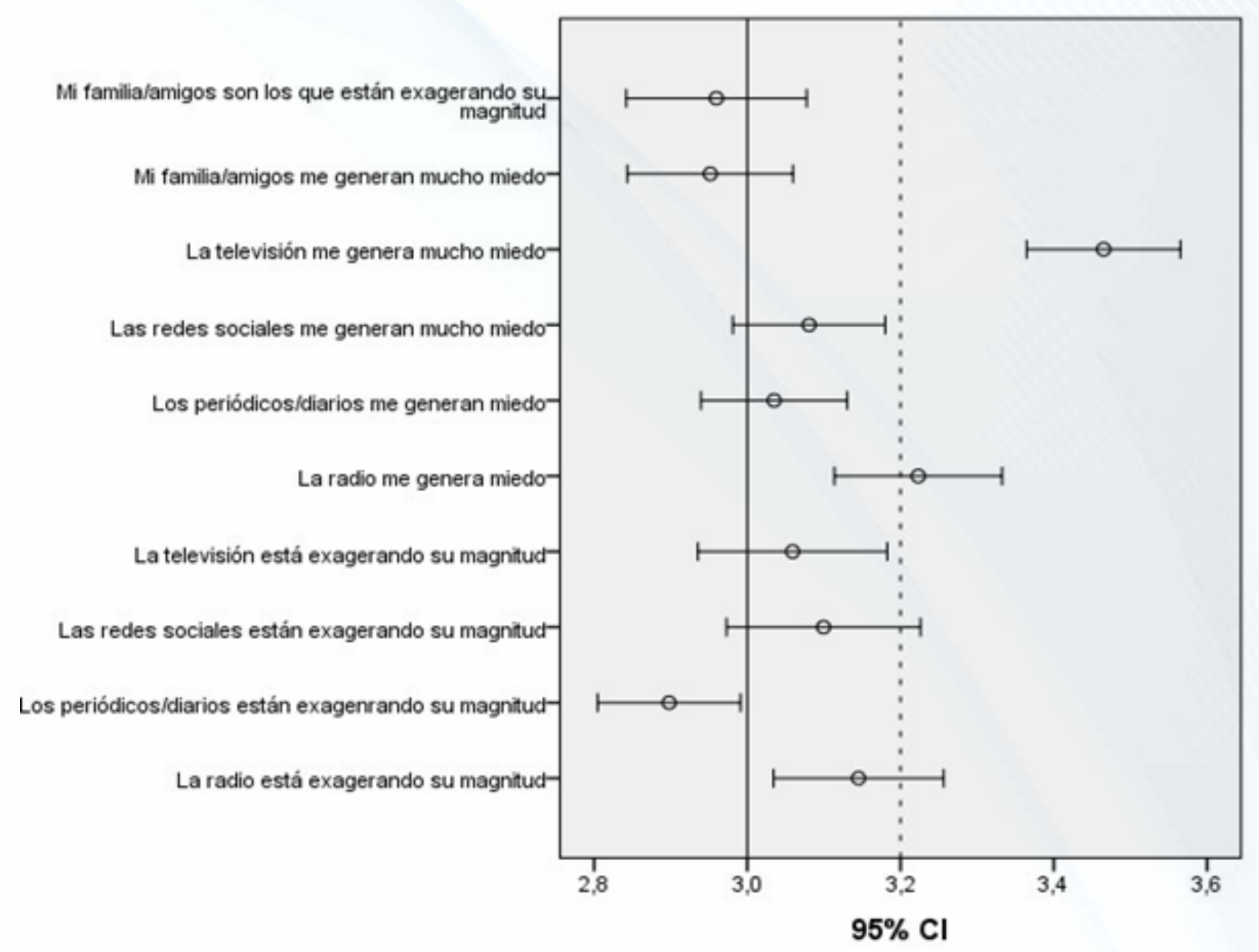

Figura 3. Comparación de las características que relacionadas al miedo y su tendencia con un intervalo de confianza del $95 \%$

En la Tabla 4, se observa la distribución de la edad $(p=0,00)$ y compañeros de trabajo $(p=0,007)$ como principales variables asociadas a la conducta en el comportamiento social de los jefes de familia. Podemos observar que la conducta inadecuada estuvo mayormente presente en los grupos de población joven o adulto y una mayor conducta adecuada en aquellos de 60 a más años. Esta diferencia fue altamente significativa $(p=0,00)$. Aquellos que percibían que el contagio no era probable a través de los compañeros de trabajo, el 75,9\% tenía un comportamiento no adecuado, pero aquellos en los cuales tenían una percepción que sí era probable el contagio a través de compañeros de trabajo, el 86,8 \% aun así tenía un comportamiento social inadecuado. Las dos condiciones inadecuadas se suman probabilísticamente. Esta diferencia fue estadísticamente significativa. Probablemente esta relación se pudo observar porque mayormente la población estuvo en condiciones de aislamiento o aislamiento parcial donde el contacto con compañeros, a pesar de considerarse como un factor de riesgo, no lo era en el mayor tiempo del día. Estas características deberán profundizarse en trabajos futuros, pues se considera una de las principales acciones asociadas a transmisibilidad en los grupos humanos con contacto. 
Tabla 4

Características más asociadas al comportamiento social

\begin{tabular}{|c|c|c|c|c|c|c|c|}
\hline \multirow{3}{*}{ Edad } & \multicolumn{7}{|c|}{ Comportamiento social } \\
\hline & \multicolumn{2}{|c|}{ No adecuado } & \multicolumn{2}{|c|}{ Adecuado } & \multicolumn{2}{|c|}{ Total } & \multirow{2}{*}{$\mathbf{p}$} \\
\hline & . & . & . & & . & . & \\
\hline De 20 a 29 & 32 & $84,20 \%$ & 6 & $15,80 \%$ & 38 & $100 \%$ & \\
\hline De 30 a 39 & 46 & $79,30 \%$ & 12 & $20,70 \%$ & 58 & $100 \%$ & \\
\hline De 40 a 49 & 130 & $92,90 \%$ & 10 & $7,10 \%$ & 140 & $100 \%$ & 0,000 \\
\hline De 50 a 59 & 66 & $82,50 \%$ & 14 & $17,50 \%$ & 80 & $100 \%$ & \\
\hline De 60 a más & 33 & $58,90 \%$ & 23 & $41,10 \%$ & 56 & $100 \%$ & \\
\hline Total & 307 & $82,50 \%$ & 65 & $17,50 \%$ & 372 & $100 \%$ & \\
\hline \multicolumn{8}{|c|}{ Probabilidad de contagio desde Compañeros de trabajo } \\
\hline No & 110 & $75,90 \%$ & 35 & $24,10 \%$ & 145 & $100 \%$ & \multirow{2}{*}{0,007} \\
\hline Sí & 197 & $86,80 \%$ & 30 & $13,20 \%$ & 227 & $100 \%$ & \\
\hline Total & 307 & $82,50 \%$ & 65 & $17,50 \%$ & 372 & $100 \%$ & \\
\hline
\end{tabular}

\section{Discusión}

Abdulkareem et al. refieren que la sociedad moderna enfrenta muchos riesgos, incluyendo enfermedades, desastres naturales y amenaza tecnológica. Se requiere una comprensión profunda de cómo las personas ven los riesgos y la efectividad de las medidas de protección. En el proceso individual o en el proceso colectivo, se presta poca atención al papel del aprendizaje inteligente en la evaluación de riesgos y la toma de decisiones de protección. La interacción social es esencial tanto para el aprendizaje individual como para el aprendizaje grupal. ${ }^{8}$ Se estudia las características fenomenológicas de cómo abordar integralmente el trabajo con la comunidad y cómo afrontar el trabajo sanitario con el individuo. Las características propias de cada lugar, grupo humano y personal explican la gran dificultad que se tuvo para afrontar esta pandemia, una de las más riesgosas aún en un actual contexto moderno y científico tal como se abordan los problemas sanitarios. Es necesario entender que cada grupo humano de cada región o conglomerado de personas mostrarán comportamientos y afrontamientos diferentes que es necesario conocer. Los resultados deberán servir para abordar eficientemente las estrategias en la comunidad. En este contexto, nuestra población de estudio manifiesta características muy particulares en la mayoría y otras que se podrían generalizar a otras sociedades. Este comportamiento social, percepción de temor o miedo y la percepción de estar en riesgo podrían explicar porque en Tacna tuvimos una "explosión" de casos que escapó al control epidemiológico con una frecuencia de muerte que superó los 600 fallecidos.

Ky et al. ${ }^{18}$ refieren que en una epidemia infecciosa la población humana puede asumir medidas preventivas en un intento de evitar infectarse, pero el comportamiento racional a nivel individual o colectivo, como el distanciamiento social, puede no siempre ser beneficioso para la población en general. Refiere que, en muchos casos, el distanciamiento social puede evitar que ocurra un brote, pero cuando el distanciamiento social es moderado puede empeorar el resultado de la enfermedad. En nuestros resultados evidenciamos que este concepto es real. En el supuesto deseado de un aislamiento total, observamos que este no se cumplió. En la muestra de estudio representativa, el 54,60\% refiere que ha cumplido con este mecanismo parcialmente y un 32,80\% prefiere no opinar al respecto. Solo el 12,70 \% confirma haber realizado, hasta la culminación del presente 
estudio, un aislamiento total. Un aislamiento moderado podría considerarse aún más peligroso o riesgoso que aquel que no se realizó puesto que oculta la real dimensión de una de las medidas evitativas más eficientes conocida hasta la fecha. En ese escenario, se recomendaría mejor el distanciamiento social más que el aislamiento.

Bavel et al. describió que la pandemia de COVID19 representa una crisis de salud global masiva y que requiere un cambio de comportamiento a gran escala. Identificó varias ideas para una respuesta efectiva a la pandemia de COVID-19 y destacaron las lagunas importantes que los investigadores tienen aún. ${ }^{22}$ Si esto es reconocido a nivel mundial, es muy necesario contar con la mayor cantidad de trabajos como el presente para conocer las verdaderas características que muestra cada grupo humano. En el contexto de comportamiento social y afrontamiento del miedo aún se conoce muy poco, y en la población sujeta de la presente investigación se logró resumir que el 34,9 \% tuvo contacto con familiar cercano pero que no habitaba en la misma casa. Un 28,2 \% refiere que supo de un amigo cercano que era COVID-19 positivo, el 19,1 $\%$, un vecino. Así como que, el $96 \%$ de la muestra en estudio usa la televisión como medio orientador, seguido de un $71 \%$ de la radio, el 60,5 $\%$ a través de medios de internet. Además de ello, solo el 43,5 \% asume que por conductas propias se considera en riesgo. El 54,60 \% refiere que ha cumplido con este mecanismo de aislamiento parcialmente y un 32,80\% prefiere no opinar al respecto. Solo el 12,70 \% confirma haber realizado un aislamiento tal como se le pidió por medios oficiales sanitarios a nivel nacional.

Mejía et al. observa en su trabajo que, en la crisis de salud mundial, los medios de comunicación son importantes para informar problemas relacionados con la población. Las redes sociales (64\%) y la televisión (57 \%) exageran la información y aumentaron el miedo. ${ }^{20}$ Es de reconocerse que no se han realizado trabajos aún a nivel regional ni local respecto al problema sujeto de estudio. Mediante nuestro trabajo se observó con un 95 \% de confianza que el uso de la televisión como instrumento orientador genera más miedo respecto a los demás medios de comunicación, dada principalmente por la exageración de la magnitud de los daños observados. Los usos de las redes estuvieron en la misma escala de riesgo de provocar temor y miedo. Hubo una diferencia estadísticamente significativa según edad y la percepción de riesgo y temor estuvo fuertemente asociada a probable transmisión de compañeros de trabajo. Sin lugar a dudas, es muy probable que el comportamiento social, especialmente de las medidas evitativas y de aislamiento, sean las principales variables a controlar en el futuro más cercano.

\section{Conclusión}

Se concluye que el 8,3\% tuvo contacto con familiar sospechoso de COVID-19 bajo el mismo techo, el $34,9 \%$ con familiar cercano y el $28 \%$ con un amigo, mientras que un 59,4\% desconoce si tuvo contacto con algún sospechoso. Y el 43,5\% percepción de contraer COVID-19 por riesgo de su propia conducta, siendo el entorno de riesgo principalmente por compañeros de trabajo ( $p=$ 0,000 ). Respecto a conducta social, el 89,5\% adopta comprar mascarillas respiratorias, el 81,70 \% opta por la adquisición de alcohol y solo el $39 \%$ adopta el lavado de manos; y como medidas evitativas, el 87,9 \% considera el uso de mascarillas de forma diaria, el $47 \%$ refiere como adecuado el evitar lugares públicos con mucha gente, el 51,10\% evitar los transportes públicos con mucha gente. Solo un 58,30 \% refirió que el evitar el contacto con personas sintomáticas es una adecuada medida evitativa. El 54,6\% de jefes de familia del cercado de Tacna ha cumplido con aislamiento parcial, el 32,80\% prefiere no opinary solo el 12,7 \% tuvo aislamiento total y permanente. Las características relacionadas al miedo fueron el uso de la televisión, la radio, uso de redes sociales y la exageración de la comunicación que en esos medios discurre. Hubo diferencia según edad y la condición de contacto con compañeros de trabajo $(p<0,01)$ asociados al miedo. El comportamiento social fue estadísticamente diferente según la edad y el contagio a partir de compañeros de trabajo $(p<$ $0,01)$

Se recomienda establecer un plan estratégico de control eficiente de regulación del aislamiento considerando la posibilidad de difundir mejor el distanciamiento social con refuerzo de medidas evitativas y de higiene. Apoyar las medidas dadas 
por el gobierno en relación a la protección social frente a la pandemia COVID-19, minimizando contacto entre compañeros de trabajo y con el cliente asegurando la distancia mínima y segura de $2 \mathrm{~m}$, garantizar sus equipos de protección personal, dar facilidades de realizar teletrabajo con el objetivo de disminuir la propagación de contagio en el trabajo velando a su vez por la salud mental del trabajador por el miedo relacionado al contagio en su trabajo. Generar un plan de asesoría a medios de comunicación para adecuados mensajes que eviten la alarma exagerada. Dirigir a los lectores a fuentes expertas y fiables, como la Organización Mundial de la Salud, contribuye a mejorar la información del público. Los medios de comunicación han de desarrollar la confianza de la audiencia en las organizaciones de la salud y profesionales expertos, para que los lectores sepan a quién recurrir para futuras recomendaciones. Fortalecer los programas de salud mental en el periodo que dure la pandemia y en las secuelas.

\section{Referencias}

1. Wu Z, McGoogan JM. Characteristics of and Important Lessons From the Coronavirus Disease 2019 (COVID-19) Outbreak in China: Summary of a Report of 72314 Cases From the Chinese Center for Disease Control and Prevention. JAMA [Internet]. 7 de abril de 2020 [citado 2 de junio de 2020];323(13):1239-42. Disponible en: https://jamanetwork.com/journals/jama/ful larticle/2762130

2. Silva DAR da, Pimentel RFW, Merces MC das, Silva DAR da, Pimentel RFW, Merces MC das. COVID-19 and the pandemic of fear: reflections on mental health. Rev Saúde Pública [Internet]. 2020 [citado 2 de junio de 2020];54. Disponible en: http =//www.scielo.br/scielo.php?script=sci_abs $\mathrm{t} r \mathrm{a} c \mathrm{t} \& \mathrm{p} i \mathrm{~d}=\mathrm{S} 0034$ $89102020000100800 \&|n g=e n \& n r m=i s o \& t| n$ $\mathrm{g}=\mathrm{en}$

3. Han Q, Curtis DR. Social Responses to Epidemics Depicted by Cinema - Volume 26, Number 2-February 2020 - Emerging Infectious Diseases journal - CDC. [citado 8 de mayo de 2020]; Disponible en: https://wwwnc.cdc.gov/eid/article/26/2/18 -1022_article
4. WOH WHO. Managing Epidemics: Key Facts About Major Deadly Diseases. World Heal Organ. 2018.

5. Morens DM, Fauci AS. Emerging Infectious Diseases: Threats to Human Health and Global Stability. Heitman J, editor. PLoS Pathog [Internet]. 4 de julio de 2013 [citado 8 de mayo de 2020];9(7):e1003467. Disponible e

https://dx.plos.org/10.1371/journal.ppat.10 03467

6. Zhao S, Wu J, Ben-Arieh D. Modeling infection spread and behavioral change using spatial games. Health Syst [Internet]. 1 de marzo de 2015 [citado 8 de mayo de 2020];4(1):41-53. Disponible en: https://doi.org/10.1057/hs.2014.22

7. Tan X, Li S, Wang C, Chen X, Wu X. Severe acute respiratory syndrome epidemic and change of people's health behavior in China. Health Educ Res [Internet]. octubre de 2004;19(5):576-80. Disponible en: https://www.ncbi.nlm.nih.gov/pubmed/15 150138/

8. Abdulkareem SA, Augustijn E-W, Filatova T, Musial K, Mustafa YT. Risk perception and behavioral change during epidemics: Comparing models of individual and collective learning. PLOS ONE [Internet]. 6 de enero de 2020 [citado 8 de mayo de $2020] ; 15(1)$. Disponible en: https://www.ncbi.nlm.nih.gov/pmc/articles /PMC6944362/

9. Abdulkareem SA, Augustijn E-W, Mustafa YT, Filatova T. Intelligent judgements over health risks in a spatial agent-based model. Int J Health Geogr. 20 de 2018;17(1):8.

10. Fonoberova M, Fonoberov VA, Mezic I. Global sensitivity/uncertainty analysis for agentbased models. Reliab Eng Syst Saf [Internet]. 1 de octubre de 2013 [citado 8 de mayo de 2020];118:8-17. Disponible en: http =//www.sciencedirect.com/science/article/ pii/S0951832013000999

11. Eberlen J, Scholz G, Gagliolo M. Simulate this! An Introduction to Agent-Based Models and their Power to Improve your Research Practice. Int Rev Soc Psychol [Internet]. 3 de julio de 2017 [citado 8 de mayo de 2020];30(1):149-60. Disponible en: http

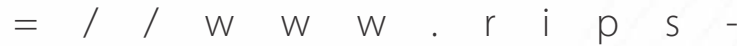
irsp.com/article/10.5334/irsp.1 
12. Samuel K. Cohn J. Epidemics: Hate and Compassion from the Plague of Athens to AIDS [Internet]. Epidemics. Oxford University Press; [citado 8 de mayo de $\left.\begin{array}{llllll}2 & 0 & 2 & 0\end{array}\right]$. Disponible e $n$

https://www.oxfordscholarship.com/view/1 0.1093/oso/9780198819660.001.0001/oso9780198819660

13. Kazanjian P. The AIDS pandemic in historic perspective. J Hist Med Allied Sci [Internet]. julio de 2014;69(3):351-82. Disponible en: https://www.ncbi.nlm.nih.gov/pubmed/23 090980

14. Covello VT, Peters RG, Wojtecki JG, Hyde RC. Risk communication, the West Nile virus epidemic, and bioterrorism: responding to the communication challenges posed by the intentional or unintentional release of a pathogen in an urban setting. J Urban Health Bull NY Acad Med [Internet]. junio de 2001;78(2):3829 1. Disponible en: https://www.ncbi.nlm.nih.gov/pubmed? $\mathrm{cmd}=\mathrm{R}$ etrieve\&db=PubMed\&list_uids=11419589\&dopt $=$ Abstract

15. Caduff C. On the Verge of Death: Visions of Biological Vulnerability. Annu Rev Anthropol [Internet]. 21 de octubre de 2014 [citado 8 de mayo de 2020];43(1):105-21. Disponible e $n$

https://www.annualreviews.org/doi/10.114 6/annurev-anthro-102313-030341

16. Eichelberger L. SARS and New York's Chinatown: the politics of risk and blame during an epidemic of fear. Soc Sci Med 1982 [Internet]. septiembre d e 2007;65(6):1284-95. Disponible e $n$ : https://www.ncbi.nlm.nih.gov/pubmed?cm $\mathrm{d}=$ Retrieve\&db=PubMed\&list_uids $=175441$ 89\&dopt=Abstract

17. Lau JTF, Kim JH, Tsui H, Griffiths S. Perceptions related to human avian influenza and their associations with anticipated psychological and behavioral responses at the onset of outbreak in the Hong Kong Chinese general population. Am J Infect Control [Internet]. febrero de 2007;35(1):38-49. Disponible e $n$ https://www.ncbi.nlm.nih.gov/pubmed?cm $\mathrm{d}=$ Retrieve\&db=PubMed\&list_uids $=172767$ 90\&dopt=Abstract

18. Ky L, F B, D S, T B. Individual preventive social distancing during an epidemic may have negative population-level outcomes. J R Soc Interface [Internet]. 1 de agosto de 2018 [citado 8 de mayo de 2020];15(145). D i s p n i ble e n : https://europepmc.org/article/PMC/61271 85

19. Kellerman. The psychological effects of isolation in protected environments | American Journal of Psychiatry [Internet]. [citado2 dejunio de 2020]. Disponible e https://ajp.psychiatryonline.org/doi/abs/10. 1176/ajp.134.5.563

20. Mejia CR, Rodriguez-Alarcon JF, Garay-Rios L, Enriquez-Anco M de G, Moreno A, HuaytanRojas K, et al. Percepción de miedo o exageración que transmiten los medios de comunicación en la población peruana durante la pandemia de la COVID-19. Rev Cuba Investig Bioméd [Internet]. 14 de abril de 2020 [citado 28 de mayo de 2020];39(2). Disponible e $n$ : $\quad h t t p$ =//www.revibiomedica.sld.cu/index.php/ib i/article/view/e698

21. Agüero Santagelo F, Nebot Adell M, Pérez Giménez A. A, López Medina MJ, García Continente X. Actitudes y comportamientos preventivos durante la pandemia de gripe (H1N1) 2009 en España. Rev Esp Salud Pública [Internet]. febrero de 2011 [citado 28 de mayo de 2020];85(1):73-80. Disponible e $\mathrm{n}$ : $\quad \mathrm{ht} \mathrm{t} \mathrm{p}$ $=/ /$ scielo.isciii.es/scielo.php?script=sci_abst $r$ a c t \& $p$ i $d=S 1135$ 57272011000100009\&lng=es\&nrm=iso\&tln $\mathrm{g}=\mathrm{es}$

22. Bavel JJV, Baicker K, Boggio PS, Capraro V, Cichocka A, Cikara M, et al. Using social and behavioural science to support COVID-19 pandemic response. Nat Hum Behav [Internet]. mayo de 2020 [citado 20 de mayo de 2020];4(5):460 Disponible e $n$ : https://www.nature.com/articles/s41562020-0884-Z

\section{Correspondencia:}

\section{jmirandab@unjbg.edu.pe}

Fecha de recepción: 12/10/2021

Fecha de aceptación:24/11/2021 